\title{
Redes de Dependencia entre Estrategias de Aprendizaje y Perfiles de Estudiantes de Desempeño Académico Medio y Alto en el Contexto de la Educación Superior en Chile
}

\author{
Yudi A. Herrera-Núñez ${ }^{(1)}$ y José A. González-Campos ${ }^{(2)}$ \\ (1) Universidad de Playa Ancha, Unidad de Acceso inclusivo y Permanencia (UAIP), Avenida Playa Ancha 850, \\ Valparaíso, Chile (e-mail: yudi.herrera@upla.cl) \\ (2) Universidad de Playa Ancha, Facultad de Ciencias Naturales y Exactas, Avenida Playa Ancha 850, Valparaíso, Chile \\ (e-mail: jgonzalez@upla.cl)
}

Recibido Sep. 25, 2018; Aceptado Dic. 4, 2018; Versión final Mar. 4, 2019, Publicado Ago. 2019

\begin{abstract}
Resumen
Se determina el tipo de estrategias de aprendizaje más empleadas por los estudiantes, de acuerdo a los niveles de desempeño académico y a algunas variables contextuales en su primer año de educación superior. Esto se hace con el objetivo de identificar perfiles en función de las redes de dependencias. La metodología empleada responde al paradigma positivista, con alcance cuantitativo, de medición transversal, en un contexto descriptivo e inferencial con una muestra aleatoria de 83 estudiantes de primer año de la Universidad de Playa Ancha (en Chile), con el uso del Cuestionario de Evaluación de Estrategias de Aprendizaje de Educación Universitaria. Los resultados nos permiten identificar dependencias significativas (5\%) con un mayor grado de explicación entre las estrategias metacognitivas, las estrategias de procesamiento y uso de información; y estrategias de control de contexto. Se caracterizan dos perfiles de estudiantes, como resultado, principalmente, del uso de las estrategias de aprendizaje metacognitivas y de procesamiento, y en menor medida respecto a las variables contextuales.
\end{abstract} dependencia; estudiantes de alto nivel de desempeño académico.

\section{Dependency Networks between Learning Strategies and Profiles of Students of Medium and High Academic Performance in the Context of Higher Education in Chile}

\begin{abstract}
The type of learning strategies most used by students, according to the levels of academic performance and some contextual variables in their first year of higher education is determined. This is done to identify profiles based on dependency networks. The methodology used in the study is based on the positivist paradigm, with a quantitative, cross-sectional measurement, in a descriptive and inferential context with a random sample of 83 first-year students of the Playa Ancha University (in Chile), with the use of the Learning Strategies Evaluation of University Education Questionnaire. Results show significant dependencies (5\%) with a greater degree of explanation among metacognitive strategies, processing strategies and use of information; and context control strategies. Two profiles of students are characterized, mainly as a result of the use of metacognitive and processing learning strategies, and to a lesser extent with respect to contextual variables.
\end{abstract}

Keywords: learning strategies; academic performance; higher education; dependency networks;

overachieving students 


\section{INTRODUCCIÓN}

La transición entre la era industrial y la del conocimiento nos exige repensar las implicaciones en cuanto a los estudios universitarios y los nuevos roles que juegan educación y aprendizaje en este desafiante contexto. Trilling y Fadel (2009) sostienen que los dos conjuntos de habilidades esenciales que permanecerán liderando la lista de requisitos laborales para el trabajo del siglo XXI son la capacidad de adquirir y emplear nuevos conocimientos y la capacidad para aplicar las habilidades esenciales, lo que exige a la educación privilegiar, por sobre la memorización, el uso de estrategias de aprendizaje. El desarrollo y los resultados de la investigación sobre calidad de la educación superior (Pérez-Luño et al., 2000) demandan la necesidad de incorporar nuevas variables que den un panorama más completo de los factores asociados al éxito o fracaso escolar en los estudios universitarios que trasciendan o se complementen las variables de orden contextual (Carvajal et al., 2018). El aprendizaje en la universidad requiere de estudiantes autónomos, capaces de gestionar nuevos aprendizajes en un contexto de conocimientos cambiantes, en cuanto a determinar sus necesidades de aprendizaje y las estrategias para adquirir estos aprendizajes (Zimmerman, 1995).

Las publicaciones referidas a investigaciones sobre estrategias de aprendizaje plantean enriquecedoras aristas para abordarlas, cuyos enfoques varían desde la implementación de programas para su enseñanza (León et al., 2014), el estudio del aprendizaje auto-regulado incluyendo factores motivacionales, cognitivos, contextuales y afectivos (Pintrich, 2003; Valencia-Serrano et al., 2013, Veas, et al., 2017 y Zimmerman, 2011), la evaluación del uso de estrategias de aprendizaje (Acevedo, et al., 2015, Gargallo et al., 2009; Román y Gallego, 1994), las relaciones entre estrategias de aprendizaje y el uso de habilidades de razonamiento y creatividad (Aizpurua et al., 2018) y la relación entre las estrategias de aprendizaje y el rendimiento académico (Gomes et al., 2014 y Muelas y Navarro, 2015). De los principales resultados de estos últimos estudios, aunque mediante el empleo de distintos instrumentos y la aplicación de diversas pruebas estadísticas, se evidencia una relación más o menos estrecha entre el uso de estrategias de aprendizaje y rendimiento académico. A pesar del interés que suscita la temática, los estudios que en Chile se han realizado con estudiantes de educación superior en torno al tema, son reducidos y hacen necesario plantearse cuestionamientos sobre el tipo de estrategias que permiten mediar el aprendizaje y mejorar los resultados académicos. En este contexto, resultó relevante preguntarse por el tipo de estrategias de aprendizaje que resultan ser más empleadas por los estudiantes de distintos niveles de desempeño académico en su primer año de educación superior e intentar identificar perfiles que evidencien esta relación junto a la incidencia de otras variables contextuales.

Monereo (1994) define las estrategias de aprendizaje como "procesos de toma de decisiones (conscientes e intencionales) en los cuales el alumno elige y recupera, de manera coordinada, los conocimientos que necesita para cumplimentar una determinada demanda u objetivo, dependiendo de las características de la situación educativa en que se produce la acción" (p. 27). Las estrategias de aprendizaje están relacionadas con la calidad del aprendizaje del estudiante, de allí que, aunque dos estudiantes compartan los mismos niveles de motivación y la misma capacidad, puedan lograr niveles de aprendizaje diferentes debido al tipo de estrategia que empleen (Pintrich, 1995). Un estudiante eficiente desarrolla un aprendizaje estratégico respecto a la gestión de (1) componentes afectivos-motivacionales que suponen la disposición y voluntad para iniciar y mantener el esfuerzo, (2) metacognición, que involucra la planificación, toma de conciencia y evaluación de los procesos propios de aprendizaje, (3) control del contexto e interacción referido al ambiente social y físico apropiado para el aprendizaje, (4) habilidades cognitivas de procesamiento de información relacionadas con la búsqueda, selección, procesamiento y transformación de la información (Gargallo et al. 2011).

En cuanto a la relación entre el uso de estrategias de aprendizaje y rendimiento académico, Muñoz, Beltrán y López (2009) caracterizan el perfil estratégico que tienen los estudiantes de alto rendimiento (con talento) en Lengua Castellana y Literatura de enseñanza media, empleando el cuestionario de estrategias cognitivas CEA cuyo perfil resultante privilegia las estrategias relacionadas con la motivación, transformación de la información, habilidades de pensamiento crítico, la planificación y regulación del aprendizaje. Cai y Zhu (2017) en un análisis expost-facto para estudiar los efectos de las estrategias de aprendizaje en la comprensión lectora en los adolescentes participantes del programa de lectura PISA chino y finlandés 2009, encuentran que las estrategias de control están relacionadas positivamente con el desempeño lector y que esta asociación se aumenta sustancialmente en co-presencia de las estrategias de memorización y de elaboración. En un contexto más cercano, Dapelo y Matus (2013) llevan a cabo un estudio en la Universidad de Playa Ancha, Chile, aplicando el Test de Estrategias de Aprendizaje: A.C.R.A (Estrategias de Adquisición, Codificación y recuperación de la información y estrategias de apoyo al procesamiento) a una muestra de 1128 estudiantes, para establecer las necesidades de fortalecimiento de las estrategias de aprendizaje en estudiantes de primer año universitario, y verificar si existen diferencias estadísticamente significativas según variables personales y contextuales. De acuerdo con sus resultados son las estrategias cognitivas y de control las que correlacionan significativamente con el rendimiento académico, además de encontrar entre las variables que diferencian el uso de estas estrategias el género, a favor de las mujeres. 
En cuanto a la identificación de perfiles de estudiantes de rendimiento académico distinto: medio y bajo respecto del uso de estrategias de aprendizaje, preocupación compartida por el presente estudio, los resultados de la literatura no son concluyentes, dada la diferencia en cuanto a sus propósitos, el empleo de distintos instrumentos de evaluación, al tamaño y selección de la muestra.

El estudio de Gargallo et al. (2011) analiza las estrategias de aprendizaje de un grupo de 148 alumnos excelentes, comparados con un grupo de alumnos medios para identificar la actuación eficaz de los primeros en términos de su rendimiento académico y mediante la aplicación del cuestionario de su autoría: CEVEAPEU. Se concluye que los alumnos excelentes usan más y mejores estrategias que los alumnos medios. Resultados proporcionalmente similares, son obtenidos por Gargallo et al (2014) quienes pretenden generar el perfil de estudiantes excelentes, con una muestra de 281 estudiantes, 148 excelentes y 133 medios. Además de contemplar el uso de estrategias de aprendizaje para determinar los perfiles, se evalúan los estilos de aprendizaje, el coeficiente intelectual y el nivel de aprendizaje, cuyos resultados son favorables en diversidad y cantidad para los estudiantes excelentes respecto del uso de Estrategias de búsqueda y selección de información ( $p<.05)$, y Estrategias de procesamiento y uso de información $(p<.05)$.

El presente trabajo tiene como objetivo principal caracterizar el perfil de los estudiantes de primer año de una universidad pública chilena, relacionando las estrategias de aprendizaje que emplean con mayor frecuencia con su rendimiento académico y algunas variables contextuales. Para alcanzar este objetivo general, se proponen los siguientes objetivos específicos: i) Jerarquizar las estrategias de aprendizaje según puntuaciones obtenidas en el instrumento CEVEAPEU; ii) Determinar si las puntuaciones obtenidas en el instrumento CEVEAPEU generan diferencias significativas entre las estrategias de aprendizaje; iii) Establecer si existe dependencia significativa entre los tipos de estrategias de aprendizaje; iv) Verificar si existe dependencia significativa entre los tipos de estrategias de aprendizaje y el rendimiento académico; y v) Caracterizar los perfiles de los alumnos considerando tipos de estrategias de aprendizaje, rendimiento académico y variables contextuales.

\section{METODOLOGÍA}

El presente estudio está enmarcado en un paradigma positivista, con alcance cuantitativo, de medición transversal, en un contexto descriptivo e inferencial, no experimental (Hernández et al., 2014), el cual incorpora un conjunto de relaciones entre preguntas de investigación e hipótesis de trabajo.

Para la caracterización del perfil de los estudiantes de primer año de la Universidad de Playa Ancha, se consideró como población a los alumnos que participan y aprueban durante el primer semestre, el programa de nivelación de competencias académicas, específicamente en el área de lenguaje, pertenecientes a la cohorte 2017, cuyo tamaño es de 130 alumnos de las carreras de Pedagogía en Educación Básica, Pedagogía en Castellano, Pedagogía en Inglés, Pedagogía en Historia y Geografía, Pedagogía en Educación Diferencial, Pedagogía en Educación Física, Bibliotecología y Administración Turística Multilingüe. La unidad de análisis es identificada con las puntuaciones obtenidas en el test CEVEAPEU. Considerando como estratos de comparación el desempeño académico, se entiende como Rendimiento académico cuantificado a través del promedio final del primer semestre y Rendimiento escolar equivalente al puntaje obtenido en la Prueba de Selección Universitaria (PSU) los resultados de la Prueba de Selección Universitaria (PSU) son requeridos para el ingreso a la educación superior en Chile y al ser un test estandarizado resulta ser un indicador relevante respecto de los aprendizajes de la enseñanza secundaria- además de variables del contexto.

Se utiliza una muestra probabilística, en la que se incluye a 83 estudiantes de las carreras señaladas, con un nivel de confianza del $95 \%$, y error de estimación del $\pm 5 \%$, según la fórmula de Namakforoosh (2000). El tipo de muestreo es aleatorio simple, esto significa que cada unidad muestral es asumida con la misma probabilidad de ser seleccionada. El tipo de muestreo indicado es a razón de la similitud del tamaño muestral en relación a la población, con una representatividad del 64\%. Para la recolección de los datos se utiliza la información proporcionada, por una parte, por la unidad de análisis institucional (caracterización sociodemográfica) y por otra, por medio de la aplicación del Cuestionario de Evaluación de estrategias de aprendizaje de Estudiantes Universitarios (CEVEAPEU) desarrollado por Gargallo et al. (2009) y que fue validado en dos universidades públicas de Valencia, España. No obstante, lo anterior, se realizó un proceso de calibración de contexto del instrumento, basados en la muestra de estudio, obteniéndose como estimador de la fiabilidad (Alpha de Cronbach) 0.77 , considerando este valor aceptable, por lo tanto, el contexto no perturba las características métricas del instrumento. Los análisis definidos fueron estimados utilizando el software R-project versión 3.4.4, particularmente Rcommander. 


\section{Descripción de las variables}

En este apartado se describe el estatus métrico de las variables de contraste para la variable respuesta, definida como puntaje obtenido en el instrumento CEVEAPEU, siendo esta cuantitativa en escala intervalar. Para el caso de las variables de contraste se tiene:

a. Rendimiento académico, entendido como la media de las calificaciones obtenidas en la universidad, cuyo estatus métrico es intervalar, con valores oscilando entre 1.0 a 7.0, siendo 1.0 la peor calificación.

b. Rendimiento Escolar entendido como la puntuación media en la prueba PSU, cuyo estatus métrico es intervalar, oscilando entre 0 a 850 puntos.

c. Desempeño Académico es una variable, cuyas categorías son identificadas con los niveles bajo, medio bajo, medio alto y alto.

d. Preferencia da cuenta de la prioridad de elección de la carrera de estudio a la cual ingresó el alumno, siendo por tanto cuantitativa y discreta, cuyos estados en orden de predilección pueden ser 1, 2, 3, etc.

e. Género es una variable dicotómica y cualitativa, cuyos estados son Femenino y Masculino.

f. Condición de Trabajo es una variable cuyos estados son caracterizados por Trabaja, Ocasionalmente o No trabaja.

g. Ingreso Económico es una variable cuantitativa de razón, en donde 0 representa la ausencia de ingreso.

h. Nivel Educacional de la Madre y Nivel Educacional del Padre es una variable categórica ordinal, cuyos estados son sin educación, educación primaria, educación primaria incompleta, educación media, educación media incompleta, etc.

\section{Descripción de la muestra}

La muestra está conformada por un $69 \%$ estudiantes de género femenino y un $31 \%$ masculino. En cuanto a la condición de trabajo de los estudiantes el $78 \%$ no trabaja, el $16 \%$ trabaja ocasionalmente y el $6 \%$ trabaja. Respecto al nivel educativo de los padres el $72 \%$ posee educación media incompleta, el $20 \%$ educación media completa, el $7 \%$ tiene formación técnica y solo el 1\% formación universitaria incompleta. Finalmente, el ingreso familiar promedio de la muestra es de 403.724 pesos chilenos.

\section{Instrumentos}

Entre los instrumentos referidos a estrategias de aprendizaje, existen pocos que incorporen las estrategias motivacionales y cognitivas. Por ejemplo, la escala LASSI (Learning and Study Strategies Inventory) es uno de los instrumentos reconocidos para evaluar las estrategias de aprendizaje; no obstante, su sistematicidad, su diseño contextualizado dificulta su utilización en el contexto chileno, pues está dirigido a alumnos estadounidenses. López (2010) respecto de la Escala CEAM II (Cuestionario de evaluación de estrategias de aprendizaje y motivación), señala que presenta desequilibrio en cuanto a los ítems, adolece de suficiente atención a los aspectos metacognitivos y estratégicos como: búsqueda, recogida y selección de la información. Por otra parte, la escala española ACRA, de Román y Gallego (1994) fue inicialmente validada en población no universitaria y su validación posterior para universitarios, no refleja su adecuación; además, Gargallo et al. (2007) señalan su descompensación dado el número de ítems que evalúa las estrategias puede oscilar entre uno y siete. Por otra parte, López (2010) si bien reconoce la sistematicidad y cuidado de esta escala, también advierte que se enfoca prioritariamente a medir técnicas y estrategias de carácter cognitivo (cognitivas, metacognitivas y de apoyo) y no tanto secuencias de procesamiento de la información como pretende.

Aunque existen distintos instrumentos para medir las estrategias de aprendizaje, en este estudio se empleó el instrumento Cuestionario de Evaluación de estrategias de aprendizaje de Estudiantes Universitarios (CEVEAPEU), dados los resultados de su proceso de calibración, el cual ha sido ajustado continuamente en rigurosas investigaciones (Gargallo et al., 2009) y distintos contextos (Bustos et al., 2017; Pegalajar, 2015), lo que ha derivado en un instrumento compensado en cuanto a la distribución de sus ítems y de características métricas deseables. Una perspectiva más amplia respecto de la comprensión de las estrategias de aprendizaje presenta Gargallo, et al. (2009), la que subyace a su propuesta del Cuestionario de Evaluación de estrategias de aprendizaje de Estudiantes Universitarios (CEVEAPEU) y es empleada para nuestro estudio. El cuestionario está organizado en dos escalas, seis tipos de subescalas y 25 estrategias de aprendizaje como se muestra en la Tabla 1. 
Tabla 1: Estructura y descripción de las subescalas del Cuestionario de Evaluación de Estrategias de Aprendizaje. (Adaptada de Gargallo et al. 2007)

\begin{tabular}{|c|c|c|c|}
\hline Escalas & Sub-escalas & Descripción & Estrategias \\
\hline \multirow{15}{*}{$\begin{array}{l}\text { Estrategias } \\
\text { afectivas, de } \\
\text { apoyo y control } \\
\text { (o automanejo) } \\
\text { (53 ítems) }\end{array}$} & \multirow{7}{*}{$\begin{array}{l}\text { Estrategias } \\
\text { motivacionales } \\
\text { (20 ítems) }\end{array}$} & \multirow{7}{*}{$\begin{array}{l}\text { Se relacionan con la manera en que el } \\
\text { estudiante maneja su disposición hacia el } \\
\text { aprendizaje, en cuanto a su motivación, } \\
\text { autoconcepto y autoeficacia }\end{array}$} & Motivación intrínseca \\
\hline & & & Motivación extrínseca \\
\hline & & & Valor de la tarea \\
\hline & & & Atribuciones internas \\
\hline & & & Atribuciones externas \\
\hline & & & Autoeficacia y expectativas \\
\hline & & & $\begin{array}{l}\text { Concepción de la inteligencia } \\
\text { como modificable }\end{array}$ \\
\hline & \multirow{2}{*}{$\begin{array}{l}\text { Componentes afectivos } \\
\text { (8 ítems) }\end{array}$} & \multirow{2}{*}{$\begin{array}{l}\text { Referidos a la relajación y control de la } \\
\text { ansiedad }\end{array}$} & Estado físico y anímico \\
\hline & & & Ansiedad \\
\hline & \multirow{4}{*}{$\begin{array}{l}\text { Estrategias } \\
\text { metacognitivas } \\
\text { (15 ítems) }\end{array}$} & \multirow{4}{*}{$\begin{array}{l}\text { Involucran el conocimiento, planificación y } \\
\text { gestión de estrategias cognitivas pertinentes, } \\
\text { facultan la toma de decisiones y la valoración } \\
\text { del propio desempeño, el control de la tarea, la } \\
\text { corrección de errores, las rectificaciones y el } \\
\text { auto-refuerzo }\end{array}$} & $\begin{array}{l}\text { Conocimiento de objetivos y } \\
\text { criterios de evaluación }\end{array}$ \\
\hline & & & Planificación \\
\hline & & & Autoevaluación \\
\hline & & & Control, autorregulación \\
\hline & \multirow{2}{*}{$\begin{array}{l}\text { Estrategias de control } \\
\text { del contexto, interacción } \\
\text { social y manejo de } \\
\text { recursos } \\
\text { (10 ítems) }\end{array}$} & \multirow{2}{*}{$\begin{array}{l}\text { Se refiere a procurar ambientes de estudio } \\
\text { adecuados, gestión del tiempo y los materiales, } \\
\text { además, del aprendizaje con pares. }\end{array}$} & Control del contexto \\
\hline & & & $\begin{array}{l}\text { Habilidades de interacción } \\
\text { social y aprendizaje con } \\
\text { compañeros }\end{array}$ \\
\hline \multirow{10}{*}{$\begin{array}{l}\text { Estrategias } \\
\text { relacionadas con } \\
\text { el procesamiento } \\
\text { de la información } \\
\text { (35 ítems) }\end{array}$} & \multirow{2}{*}{$\begin{array}{l}\text { Estrategias de búsqueda } \\
\text { y selección e información } \\
\text { (8 ítems) }\end{array}$} & \multirow{2}{*}{$\begin{array}{l}\text { Incorpora el conocimiento, discriminación y } \\
\text { conocimiento de criterios para elegir la } \\
\text { información necesaria }\end{array}$} & $\begin{array}{l}\text { Conocimiento de fuentes y } \\
\text { búsqueda de información }\end{array}$ \\
\hline & & & Selección de información \\
\hline & \multirow{8}{*}{$\begin{array}{l}\text { Estrategias de } \\
\text { procesamiento y uso de } \\
\text { la información } \\
\text { (27 ítems) }\end{array}$} & \multirow{8}{*}{$\begin{array}{l}\text { Involucra el conocimiento de técnicas para } \\
\text { adquirir la información (toma de apuntes, } \\
\text { lectura, etc.), técnicas para reestructurar y } \\
\text { organizar la información (mapas conceptuales, } \\
\text { etc), recodificar (resúmenes), pensamiento } \\
\text { crítico, recuperación de la información (técnicas } \\
\text { de recuerdo) y estrategias de uso de la } \\
\text { información para tareas académicas. }\end{array}$} & Adquisición de información \\
\hline & & & Elaboración \\
\hline & & & Organización \\
\hline & & & $\begin{array}{l}\text { Personalización y creatividad, } \\
\text { pensamiento crítico }\end{array}$ \\
\hline & & & $\begin{array}{l}\text { Almacenamiento. } \\
\text { Memorización. Uso de } \\
\text { recursos mnemotécnicos }\end{array}$ \\
\hline & & & $\begin{array}{l}\text { Almacenamiento. Simple } \\
\text { repetición }\end{array}$ \\
\hline & & & $\begin{array}{l}\text { Transferencia. Uso de la } \\
\text { información }\end{array}$ \\
\hline & & & $\begin{array}{l}\text { Manejo de recursos para usar } \\
\text { la información adquirida }\end{array}$ \\
\hline
\end{tabular}

La investigación resguarda los datos personales en coherencia con el protocolo ético de investigación, informado durante el proceso de recolección de datos, además se presentan los resultados de forma genérica, de manera de asegurar el anonimato y confidencialidad de la misma.

\section{RESULTADOS}

\section{Jerarquización y diferenciación significativa de las subescalas}

Para establecer el orden jerárquico entre las puntuaciones de cada una de las estrategias de aprendizaje, se consideró la media de las puntuaciones de cada una de ellas y luego, de manera descriptiva se ordenaron en forma descendente como se muestra en el gráfico de la Fig. 1. Para decidir respecto de la significatividad de las diferencias, se desarrolló un test de normalidad para especificar el procedimiento inferencial de comparación (Casella, et al. 2002), no siendo rechazada la hipótesis de normalidad (test de Shapiro-Wilk, W= 0.9863, p- value= .64), por lo tanto, las comparaciones se realizan utilizando técnicas paramétricas particularmente la prueba t de student para muestras pareadas, considerando como criterio de decisión el p-valor y un nivel de significancia del $5 \%$. En este proceso se caracterizan tres grupos, entre los cuales se obtienen diferencias significativas que permiten ordenar de manera jerárquica. En primer lugar, las subescalas de control de contexto, interacción social y manejo de recursos junto a las subescalas motivacionales caracterizan el primer grupo, obteniéndose puntuaciones en ellos significativamente mayores que las estrategias de procesamiento y uso de información que junto a las metacognitivas caracterizan el segundo grupo ( $p$-value $=.00, t=3.2532$ y tamaño del efecto de 
0.1481), finalmente, estas últimas poseen puntuaciones significativamente mayores que las estrategias de búsqueda y selección de información junto a componentes afectivos ( $p$-value $=.00, t=4.8516$ y tamaño del efecto de 0.2309).

$$
\begin{aligned}
& \text { Estrategias de control } \\
& \text { de contexto, } \\
& \text { interacción social y } \\
& \text { manejo de recursos } \\
& \text { (p. media= 3.9) }
\end{aligned}
$$

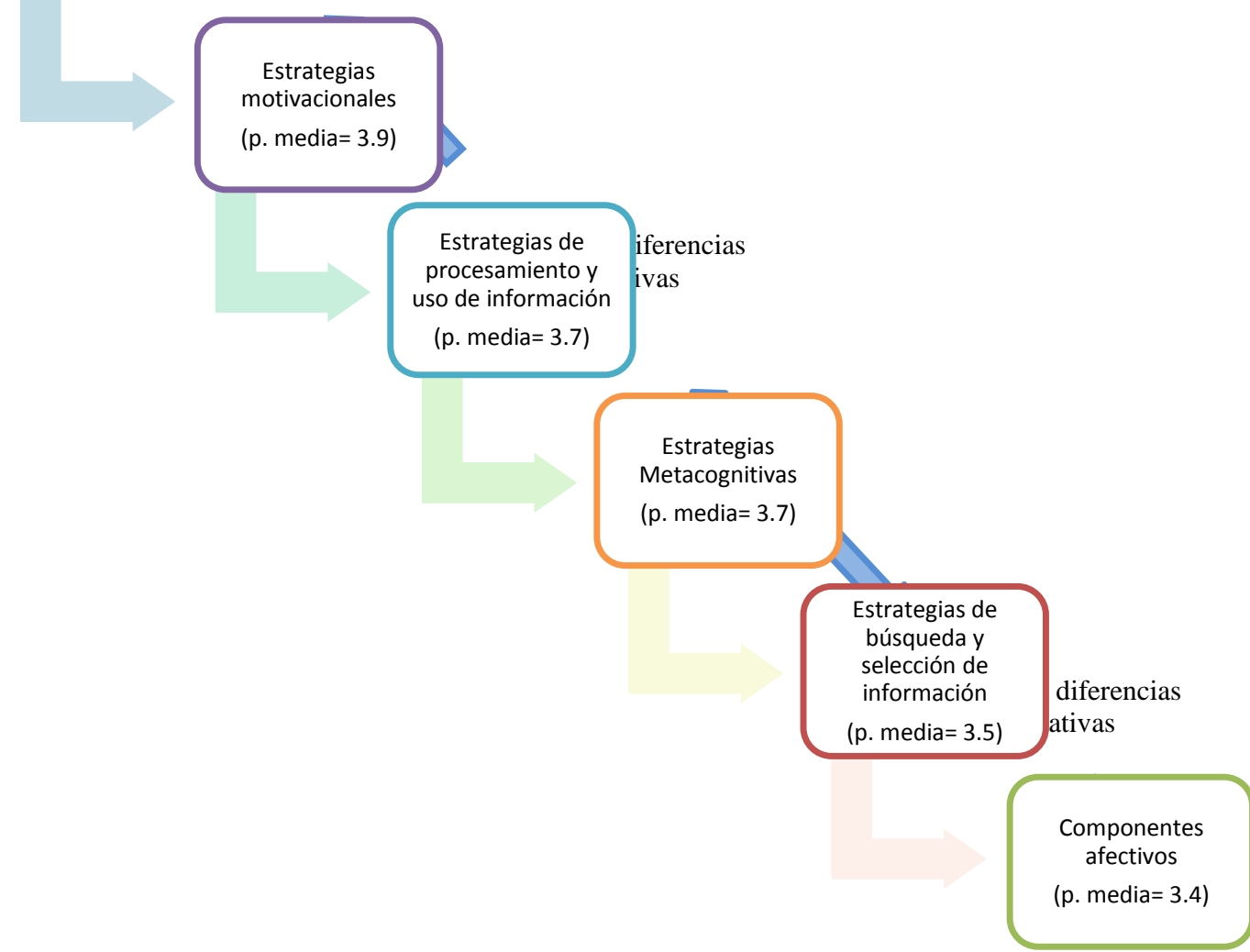

Fig. 1: Jerarquización de las subescalas de estrategias de aprendizaje según puntuación media.

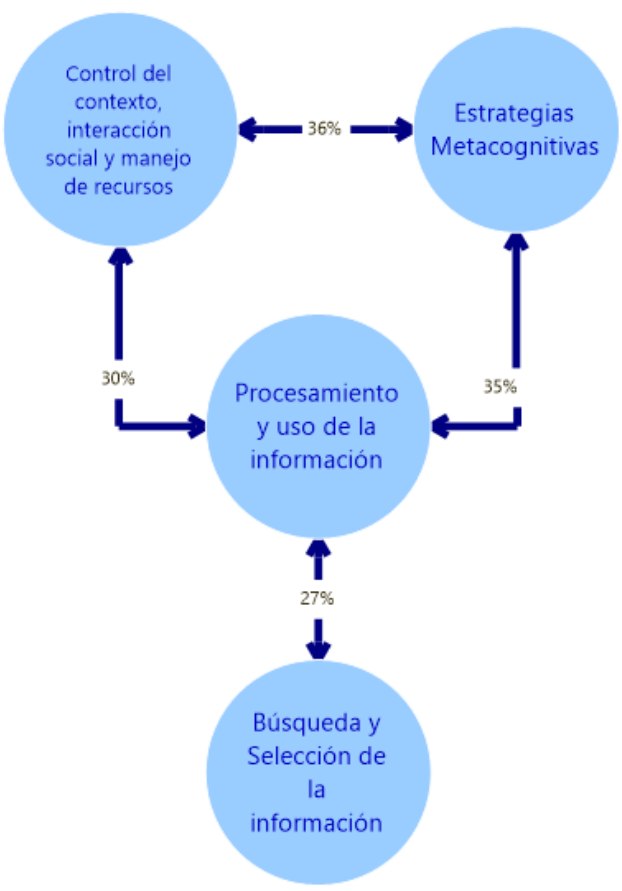

Fig. 2: Diagrama de dependencias predominantes entre subescalas de estrategias de aprendizaje. 


\section{Dependencias porcentuales entre las subescalas}

Para dar respuesta a este objetivo, se determinó la matriz de correlaciones y se seleccionaron las dependencias cuyo nivel de explicación de una sobre la otra fuese superior al 25\% (ver Fig. 2). En este proceso se determinaron las siguientes dependencias: 1. Entre las subescalas estrategias de búsqueda y selección de información, y de procesamiento y uso de información, existe una dependencia lineal, directa y significativa, con un coeficiente de correlación de Pearson 0.52, lo que significa en términos porcentuales que la variabilidad de una es explicada por la otra en un $27 \%(p$-value $=.00)$. 2. Entre las subescalas estrategias metacognitivas y de procesamiento y uso de información, existe una dependencia lineal, directa y significativa, con un coeficiente de correlación de Pearson 0.59 , lo que significa en términos porcentuales que la variabilidad de una es explicada por la otra en un $35 \%$ ( $p$-value $=.00)$. 3. Entre las subescalas estrategias de procesamiento y uso de información y las de control de contexto existe una dependencia lineal, directa y significativa, con un coeficiente de correlación de Pearson 0.55 , lo que significa en términos porcentuales que la variabilidad de una es explicada por la otra en un $30 \%(p$-value $=.00)$. 4. Entre las subescalas de estrategias metacognitivas y las de control de contexto, interacción social y manejo de recursos existe una dependencia lineal, directa y significativa, con un coeficiente de correlación de Pearson 0.60 , lo que significa en términos porcentuales que la variabilidad de una es explicada por la otra en un $36 \%$ ( $p$-value $=.00)$.

\section{Redes de dependencias estrategias de aprendizaje y desempeño académico}

Para analizar la dependencia entre las estrategias de aprendizaje y el desempeño académico — entendido como el promedio de notas del primer semestre de educación superior (R. académico) y el resultado de la PSU tomada al egreso de la enseñanza secundaria (R. escolar)-, se procedió a construir una red de dependencias.

Para la comprensión de las redes conceptuales de la Fig. 3 y Fig. 4, se debe indicar que cada nodo o vértice representa una subescala de las estrategias de aprendizaje, y cada una de las aristas o líneas representa la existencia de vínculo o dependencia entre los nodos respectivos. Este tipo de vínculo existe cuando la dependencia entre los nodos alcanza un nivel significativo, en este caso cuando es superior al $15 \%$. Por ejemplo, si los nodos Sub-escalas Metacognitivas y Componentes Afectivos se encuentran conectados por medio de una arista, entonces se puede afirmar que un $15 \%$ de la variabilidad del uso de las subescalas Metacognitivas es explicada por Componentes Afectivos. El porcentaje de explicación de una variable sobre la otra fue estimado por medio del coeficiente de determinación, por tanto, en términos correlacionales se habla de dependencias lineales directas e inversas por sobre 0.39 puntos según el coeficiente de correlación de Pearson, como se muestra en el gráfico de la Fig. 3.

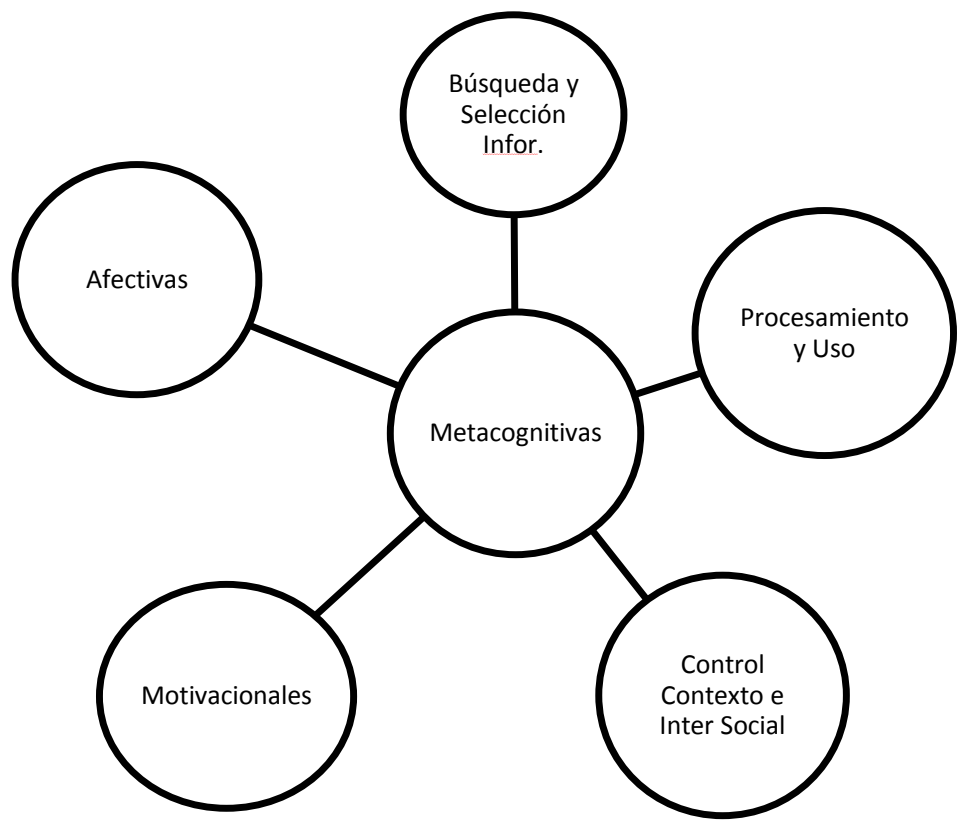

Fig. 3: Red de dependencias entre las subescalas de estrategias de aprendizaje y el desempeño Académico.

Red de dependencias entre las subescalas de aprendizaje y el desempeño académico

Las relaciones entre los indicadores de desempeño académico (R. Académico y R. Escolar), en la muestra estudiada, no evidencian una relación de dependencia con ninguna de las seis subescalas de estrategias de aprendizaje. No obstante, el rendimiento académico está gradualmente más cercano a la subescala de 
Procesamiento y uso de la información. Asimismo, es destacable la posición concéntrica de la subescala de estrategias metacognitivas respecto de las otras, como se observa en Fig. 3. Dada la homogeneidad de los resultados de desempeño académico —en cuanto al Rendimiento académico y escolar-, para efectuar el análisis de dependencias de acuerdo al perfil, se generaron solo dos grupos de desempeño académico: Nivel Medio y Nivel Alto, cuyos resultados se presentan en la Fig. 4.

Red de dependencias entre estrategias de aprendizaje

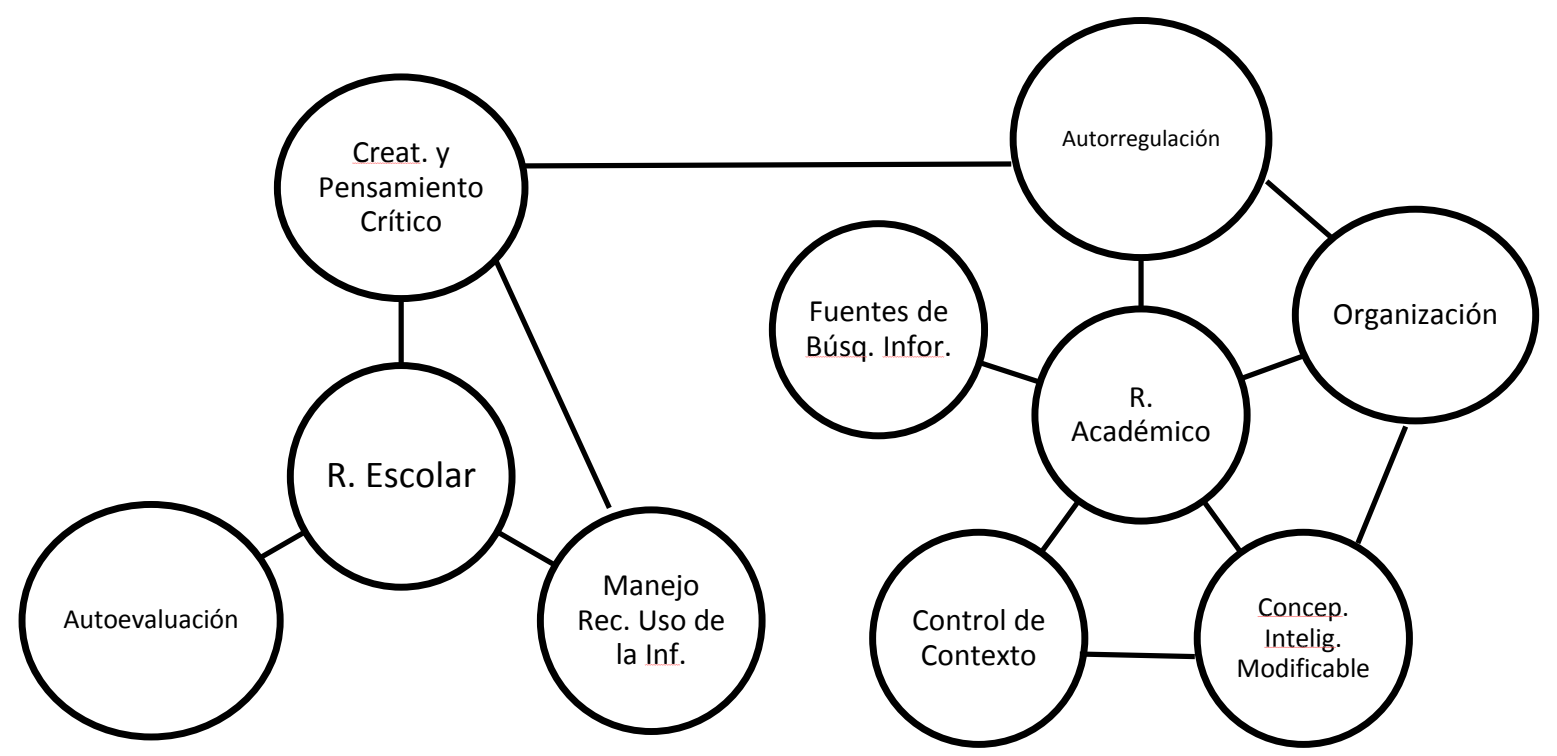

Fig. 4: Red de dependencias entre las estrategias de aprendizaje y el nivel medio de desempeño académico.

Los resultados del desempeño académico de los estudiantes de nivel medio (Fig. 4), evidencian una dependencia respecto del uso de las subescalas de las estrategias de aprendizaje de: a) Rendimiento escolar respecto de las estrategias de Autoevaluación (S. Metacognitiva) y Manejo de recursos, Personalización y creatividad, pensamiento crítico (S. Procesamiento y Uso de la información) b) Rendimiento académico evidencia una relación de dependencia con todas las subescalas y con las siguientes estrategias: Organización, Manejo de recursos y Mnemotécnia (S. Procesamiento y uso de la información); Autorregulación (S. Metacognitiva); Concepción de la inteligencia modificable (S. Motivacional), Control del contexto (S. Control del contexto e interacción social) y Fuentes de búsqueda de información (S. Estrategias de búsqueda y selección e información).

Red de dependencias entre estrategias de aprendizaje para nivel alto
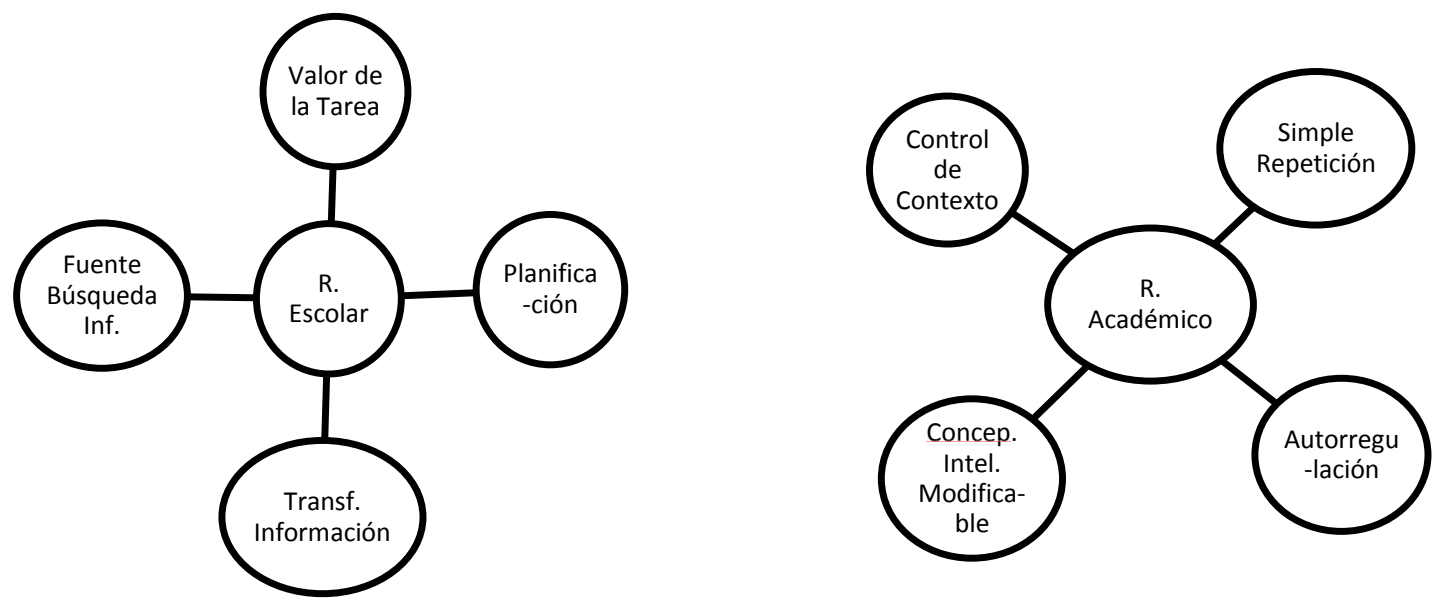

Fig. 5: Red de dependencias entre las estrategias de aprendizaje y el nivel alto de desempeño académico.

Los resultados del desempeño académico de los estudiantes de nivel alto, muestran una dependencia respecto del uso de las estrategias de aprendizaje (ver Fig. 5) en relación al: a) Rendimiento escolar respecto de las estrategias: Valor de la tarea (S. Motivacional), Planificación (S. Metacognición), Transferencia uso de la Información, Manejo de recursos y Uso de la información (S. Procesamiento y Uso de la información) y 
Fuente y búsqueda de información (S. Estrategias de búsqueda y selección e información ); b) Rendimiento académico evidencia una relación de dependencia con todas las subescalas y con las estrategias: Simple repetición, Adquisición de la información (S. Procesamiento y uso de la información); Autorregulación, Conocimiento de objetivos (S. Metacognitiva); Concepción de la inteligencia modificable (S. Motivacional) y Control del contexto (S. Control del contexto e interacción social).

\section{Verificación de dependencias significativas}

Para responder a este objetivo, se procedió a ajustar un modelo de regresión lineal, especificando como predictores cada una de las seis subescalas de estrategias de aprendizaje y como respuesta, la variable rendimiento académico. En este procedimiento se concluye que no existe relación lineal entre las estrategias de aprendizaje y rendimiento académico, cuyo nivel máximo de explicación alcanza el $2 \%$ lo que no es significativo $(p$-value $=.23$ ). Para sobreponerse a posibles problemas de colinealidad, se realizó una regresión lineal por cada uno de los predictores, sin embargo, no alcanzan un nivel de asociación significativo.

\section{Perfiles de los alumnos y uso de subescalas de estrategia de aprendizaje}

El perfil de los alumnos de la muestra, fue organizado en cuatro niveles subdividiendo en dos los niveles de desempeño académico en bajo, medio bajo (nivel medio de las redes) y medio alto y alto (nivel alto de las redes) como es descrito en la Tabla 2. Tomando como referencia el estudio descriptivo tras aplicar el Cuestionario CEVAPEAU, podemos identificar cuatro perfiles que vinculan el rendimiento académico, organizados en estos niveles: Desempeño académico Bajo, Medio Bajo, Medio Alto y Alto que realizan un uso más frecuente de las siguientes estrategias de aprendizaje y se caracterizan de la siguiente manera:

Tabla 2: Caracterización de los perfiles de los estudiantes según frecuencia de uso de Sub-escala de Estrategia de Aprendizaje

\begin{tabular}{|c|c|c|c|c|c|}
\hline & Desempeño académico & Bajo & Medio bajo & Medio alto & Alto \\
\hline \multirow{5}{*}{ 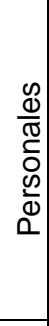 } & $\begin{array}{l}\text { Rendimiento académico } \\
\text { (prom semestre) }\end{array}$ & 3,9 & 4,9 & 5,9 & 6,7 \\
\hline & Rendimiento escolar/psu & 538,5 & 542,9 & 547,1 & 572,5 \\
\hline & Estrategia predominante & $\begin{array}{l}\text { S. Procesamiento y uso } \\
\text { de la información }\end{array}$ & $\begin{array}{l}\text { S. Estrategias } \\
\text { Motivacionales }\end{array}$ & $\begin{array}{c}\text { Estrategias } \\
\text { Motivacionales } \\
\end{array}$ & $\begin{array}{l}\text { S. Estrategias } \\
\text { Metacognitivas } \\
\end{array}$ \\
\hline & Preferencia & 1 & 2 & 2 & 1 \\
\hline & Género & Masculino & Femenino & Femenino & Femenino \\
\hline \multirow{4}{*}{ 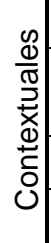 } & Trabaja & Ocasionalmente & No & No & No \\
\hline & Ingreso-econ & $\begin{array}{l}\$ 456.000- \\
\$ 570.000 \\
\end{array}$ & $\begin{array}{l}\$ 262.000- \\
\$ 345.000\end{array}$ & $\begin{array}{l}\$ 445.000- \\
\$ 456.000\end{array}$ & $\begin{array}{l}\$ 456.000- \\
\$ 570.000 \\
\end{array}$ \\
\hline & N.E. madre & Media completa & Media completa & Media incompleta & $\mathrm{S} / \mathrm{i}$ \\
\hline & N.E. padre & Media completa & Media completa & Media incompleta & $\mathrm{S} / \mathrm{i}$ \\
\hline
\end{tabular}

Tal y como se aprecia en la Tabla 2, los resultados indicarían que los estudiantes de primer semestre de la Universidad de Playa Ancha, poseen un desempeño académico coherente con los resultados de su Rendimiento escolar (PSU), es decir, una mejor puntuación en la PSU se condice con un mejor logro en el rendimiento académico del primer semestre (promedio de notas), los que junto a las variables personales de los estudiantes configuran cuatro perfiles de estudiantes; i. Nivel alto: corresponde a un estudiante de desempeño académico alto en comparación con los otros perfiles, tanto en los resultados de la Prueba de Acceso a la Educación superior (PSU), como en el promedio general del semestre (RA). Este estudiante emplea con mayor frecuencia la subescala estrategias metacognitivas de aprendizaje y posee una clara preferencia (orientación vocacional) por estudiar la carrera que ha elegido (preferencia1).

En cuanto a los rasgos contextuales, los ingresos familiares están en un rango de medio bajo, esto es entre 496 y 570 mil pesos ii. Nivel Medio-alto: este perfil está configurado por características personales de estudiantes con un promedio de rendimiento académico de 59, una preferencia de carrera menos clara (Preferencia 2) y por emplear más frecuentemente la subescala de estrategias motivacionales. En cuanto a las características contextuales, el nivel de escolaridad de los padres es media incompleta. Este rasgo resulta congruente con los resultados del estudio sobre variables predictivas de la deserción de estudiantes de Ciencias de la misma universidad de Carvajal et al., (2018) quienes advierten efectos negativos del incremento de la variable nivel educativo del padre respecto de la probabilidad de desertar, lo que en este estudio se traduce en que los estudiantes de mayor desempeño académico tienen padres con menor nivel educativo, y por ende, presentan menor riesgo de deserción, puesto que este estudiante corresponde a la primera 
generación en acceder a la universidad iii. Nivel Medio bajo: Sus características son similares al perfil anterior, caracterizándose por un rendimiento académico en promedio de 4.9, cuya subescala de estrategia de aprendizaje más empleada es la motivacional. El nivel escolar de los padres es educación media completa. iv. Nivel bajo: muestra un menor desempeño académico, un uso más frecuente de estrategias de procesamiento y uso de la información, con una clara preferencia de elección de carrera (preferencia1), trabajo ocasional y padres con formación media completa.

\section{DISCUSIÓN Y ANÁLISIS}

Las investigaciones que abordan el uso de las estrategias de aprendizaje de los estudiantes universitarios, a la vez han integrado el estudio de los enfoques de enseñanza, los estilos de aprendizaje y el nivel de aprendizaje. Todos o algunos de ellos relacionados con el rendimiento académico para evaluar en qué medida pueden predecirlo o caracterizar grupos de estudiantes de distinto nivel de desempeño. Los resultados de este estudio y decisiones sobre las hipótesis consideran el p-valor como factor decisivo, se sintetizan a continuación. Los resultados de esta investigación nos permiten concluir que las estrategias de aprendizaje no son un predictor significativo del rendimiento académico, esto quiere decir que no existe dependencia significativa entre estas variables. Siendo este resultado poco convergente con la evidencia teórica y empírica de otros estudios mencionados en el marco teórico (Gomes et al., 2014 y Muelas y Navarro, 2015).

Los datos dan evidencia de la configuración de dos perfiles de estudiantes tomando en consideración el rendimiento académico, uno caracterizado por un rendimiento bajo y medio bajo; y otro, por un rendimiento medio alto y alto, sumado a las variables contextuales; pese a ello las diferencias observadas no resultan ser estadísticamente significativas. En cuanto a los estudiantes de desempeño alto, los resultados del presente estudio, identifican el uso de una variedad de estrategias de aprendizaje: control del contexto (S. Control del contexto e interacción social), autorregulación, conocimiento de los objetivos (S. Metacognitiva), adquisición de la información, simple repetición (S. Procesamiento y uso de la información) y selección de información (S. Búsqueda y selección de la información). Cabe considerar que no se vincula a este perfil ninguna estrategia perteneciente a la subescala componentes afectivos, ni motivacionales; a diferencia de los resultados de los estudios antes mencionados (Gargallo et al., 2011, 2014 y Muñoz et al., 2009). En este último estudio, se encuentra diferencias significativas en la subescala componentes afectivos en relación al rendimiento académico de los estudiantes de alto desempeño. Sin embargo, en los resultados del presente estudio, sólo al flexibilizar el porcentaje de dependencia, no inferior al 15\%, estas pueden ser observadas interactuando con la subescala de estrategias metacognitivas.

Eso implicaría que el perfil del estudiante de rendimiento académico alto, según los resultados del presente estudio, es un estudiante caracterizado principalmente, por el uso y la interinfluencia de las subescalas de Estrategias Metacognitivas y Procesamiento y uso de la información, las que, para este estudio, resultan mutuamente dependientes $\left(\mathrm{R}^{2}=.35, \mathrm{p}\right.$-value $\left.=.00\right)$. El estudiante de alto de desempeño se caracteriza por un uso variado de las estrategias de la S. Estrategias metacognitivas, un uso frecuente de autorregulación, es decir, conciencia de autodirección, conocimiento y control de su propio proceso, de sus necesidades de aprendizaje y capacidad de planificación para resolverlas. En los estudios antes citados, la dimensión metacognitiva caracteriza, también, el perfil de estudiantes excelentes o talentosos. En cuanto a Procesamiento y uso de la información se consideran estrategias eficaces en el aprendizaje (Cai y Zhu, 2017; Gargallo et al., 2014 y Muñoz et al., 2009). Los estudiantes de desempeño alto, las emplean en el proceso de adquirir información y la activación del conocimiento previo para generar aprendizaje favoreciendo estrategias de recuperación de la información aprendida, para en algunos casos lograr su transformación. Estas subescalas están presentes en los perfiles de estudiantes de rendimiento alto y medio, solo que privilegian el uso de distintas estrategias. Asimismo, Veas, et al. (2017) en su estudio aportan evidencia sobre tres variables que muestran un nivel importante de predicción del rendimiento académico, específicamente aquellas relacionadas con las estrategias de aprendizaje (elaboración, metacognición y estrategias de personalización) y se utilizaron en menor medida por los estudiantes de bajo rendimiento, en su caso.

Respecto de la subescala Control del contexto e interacción social, los estudiantes de alto desempeño académico de este estudio, al igual que en la caracterización del perfil de los estudiantes excelentes de Gargallo, et al $(2011,2014)$, presentan una diferencia significativa en la estrategia (Control del contexto, $\mathrm{p}=.05$ ). Resultados proporcionalmente coincidentes con la caracterización del uso de estrategias de aprendizaje de los estudiantes de la Universidad de Playa Ancha, realizada por Dapelo y Matus (2013), en dónde se señala con cautela el uso frecuente que realizan estudiantes de buen rendimiento académico de las estrategias de apoyo. Esto significa para los resultados de este estudio, que el perfil de los estudiantes de alto desempeño académico de esta Universidad se identifica con la frecuencia por el uso de estrategias de aprendizaje con pares, aprendizaje colaborativo, y la habilidad de solicitar y dar apoyo en el proceso de aprendizaje. A diferencia, de los resultados de Pegalajar (2015) quien, aplicando el mismo instrumento, no encuentra diferencias significativas para esta subescala. Aizpurua et al. (2018) al analizar las estrategias de 
aprendizaje y las diferencias de su uso en habilidades de razonamiento y creatividad, concluyen que los estudiantes con mayores niveles de razonamiento fluido y científico reportan un mayor uso de estrategias de control del contexto. Lo que contribuye a explicar en nuestros resultados, el papel de este tipo de estrategias en la formación de habilidades de razonamiento científico que podrían desembocar en un mejor desempeño académico.

En cuanto a las estrategias de motivación, en el perfil de desempeño alto, su presencia implica su interés por aprender y la persistencia en la tarea (que impide el abandono), aunque la literatura plantea la motivación intrínseca como característica de los estudiantes de desempeño alto (Muñoz, et al., 2009) en nuestro estudio esta subescala no está vinculada estrechamente con este perfil; aunque sí con los estudiantes de desempeño medio y medio bajo. Los resultados del estudio de García-Ros et al. (2018) son coincidentes en cuanto a establecer que las estrategias de aprendizaje tanto de motivación, como de interacción social tienen efectos indirectos sobre el rendimiento y, por ende, en la permanencia a través de las experiencias universitarias de primer año.

\section{CONCLUSIONES}

La diversidad de los resultados de los estudios discutidos, incluido el presente, confirman la necesidad de profundizar en el uso de estrategias de aprendizaje y su incidencia en el desempeño académico, en términos de los resultados, es interesante que no exista dependencia significativa entre estas variables, lo que da lugar a nuevas preguntas de investigación. Si bien, se logra identificar dos grupos de estudiantes, uno caracterizado por poseer un rendimiento bajo y medio bajo; y el otro, por un rendimiento medio alto y alto; no se evidencian diferencias estadísticamente significativas entre ambos grupos. Los resultados nos permiten señalar que existen dependencias significativas $(5 \%)$ con un mayor grado de explicación entre las estrategias metacognitivas y las estrategias de procesamiento y uso de información; así como también, con las estrategias de control de contexto, interacción social y manejo de recursos. Se identificaron dependencias significativas de menor grado de explicación entre las estrategias de búsqueda y selección de información, y las estrategias de procesamiento y uso de información; y entre las estrategias de procesamiento y uso de información y las estrategias de control de contexto.

Resulta importante para este estudio, reconocer la centralidad de las estrategias metacognitivas y de uso y procesamiento de la información; y en el caso particular de esta comunidad educativa, de las estrategias de control de contexto, interacción social y manejo de recursos respecto del desempeño académico.

\section{REFERENCIAS}

Acevedo, D., M. Durán y A. Alvis, Identificación de Estrategias de Aprendizaje de Estudiantes de Ingeniería de Alimentos en los Cursos Balance de Materia, doi: 10.4067/S0718-50062015000600005, Formación Universitaria, 8(6), 31-38 (2015)

Aizpurua, A., L. Izarne e I. Idoia, Learning Strategies and Reasoning Skills of University Students, doi: 10.1016/j.psicod.2018.01.001, Revista de Psicodidáctica 23 (2), 110-116 (2018)

Bustos, V., A. Oliver, L. Galiana y P. Sancho, Propiedades Psicométricas del CEVEAPEU: Validación en Población Peruana, doi: 10.5944/educxx1.17513, Educación XX1, 20(1), 299-318 (2017)

Cabrera, L., J. Bethencourt, M. González y P. Álvarez, Un Estudio Transversal Retrospectivo sobre Prolongación y Abandono de Estudios Universitarios, ISSN: 1134-4032, RELIEVE. Revista Electrónica de Investigación y Evaluación Educativa, 12(1), 105-127 (2006)

Cai, Y. y X. Zhu, Learning Strategies and Reading Literacy among Chinese and Finnish Adolescents: Evidence of Suppression, ISSN:0144-3410, Educational Psychology, 37:2, 192-204 (2017)

Carvajal, C., J. González y S. Sarzoza, Variables Sociodemográficas y Académicas Explicativas de la Deserción de Estudiantes en la Facultad de Ciencias Naturales de la Universidad de Playa Ancha (Chile), doi: 10.4067/S071850062018000200003, Formación Universitaria 11(2), 3-12 (2018)

Casella, G. y R. Berger, Statistical Inference, 2, 467-492, Pacific Grove, Duxbury, Canadá (2002)

Dapelo, B. y M. Matus, Necesidades de Fortalecimiento Cognitivo-Motivacional en Estudiantes Universitarios de Primer Año: Implicaciones para una Nivelación Efectiva de Competencias, Revista de Orientación Educacional, 27(52), 15-33 (2013)

García-Ros, R., F. Pérez-González, F. Cavas-Martínez y J. M. Tomás, Social Interaction Learning Strategies, Motivation, First-Year Students' Experiences and Permanence in University Studies, doi:10.1080/01443410.2017.1394448, Educational Psychology, 38:4, 451-469 (2018)

Gargallo, B. y J. Suárez, Una Aproximación al Perfil de los Estudiantes Universitarios Excelentes, doi: https://doi.org/10.4995/redu.2014.5643, Revista de Docencia Universitaria, 12(2), 143-165 (2014)

Gargallo, B., G. Almerich y otros tres autores, Aprendizaje Estratégico en Estudiantes Universitarios Excelentes y en Estudiantes Medios, ISSN: 2340-6577, Bordón, Revista de Pedagogía 63(4), 43-64 (2011) 
Gargallo, B., J. Suárez y A. Ferreras, Estrategias de Aprendizaje y Rendimiento Académico en Estudiantes Universitarios, ISSN: 0212-4068, Revista de Investigación Educativa, 25 (2), 421-441 (2007)

Gargallo, B., J. Suárez y C. Pérez, El cuestionario CEVEAPEU. Un Instrumento para la Evaluación de las Estrategias de Aprendizaje de los Estudiantes Universitarios, E-ISSN: 1134-4032, RELIEVE, 15, 1-31 (2009)

Gomes, C., H. Golino e I. Menezes, Predicting School Achievement Rather than Intelligence: Does Metacognition Matter? doi: 10.4236/psych.2014.59122, Psychology, 5, 1095-1110 (2014)

Hernández, R., C. Fernández y L. Baptista, Metodología de la Investigación, 6ª Ed., McGraw-Hill Education, México D.F., México (2014)

León, A.P., E. Risco del Valle y C. Alarcón, Estrategias de Aprendizaje en Educación Superior en un Modelo Curricular por Competencias, doi: 10.1016/j.resu.2015.03.012, Revista de la Educación Superior, 43(172) 123-144 (2014)

López, M., Diseño y Análisis del Cuestionario de Trabajo Autónomo (CETA) para Estudiantes Universitarios, ISSN: 11361034, Revista de Psicodidáctica, 15(1), 77-99 (2010)

Monereo. C., (Coord.) Estrategias de Enseñanza y Aprendizaje. Formación del Profesorado y Aplicación en la Escuela. Graó, Barcelona, España (1994)

Muelas, A. y E. Navarro, Learning Strategies and Academic Achievement, doi: 10.1016/j.sbspro.2014.12.625, Procedia, Social and Behavioral Sciences, 165, 217-221 (2015)

Muñoz, P., J. Beltrán y E. López, Perfil en Estrategias de Aprendizaje de Estudiantes de Alto Rendimiento en Lengua Castellana y Literatura, ISSN: 1136-8136, Faisca, 14(16), 16-75 (2009)

Namakforoosh, M., Metodología de la Investigación, Limusa, México D.F., México (2000)

Pegalajar, M., Desarrollo de Estrategias de Aprendizaje en el Proceso de Formación Docente, ISSN: 1022-6508, Revista Iberoamericana de Educación, 68(2), 173-190 (2015)

Pérez-Luño, A., J. Ramón y J. Sánchez, Análisis Exploratorio de las Variables que Condicionan el Rendimiento Académico, ISSN: 2255-453X, Sevilla, Universidad Pablo de Olavide (2000)

Pintrich, P., A Motivational Science Perspective on the Role of Student Motivation in Learning and Teaching Contexts, ISSN-0022-0663, Journal of Educational Psychology, 95(4), 667-686 (2003)

Pintrich, P., Understanding Self-regulated Learning, 3-35, San Francisco, Jossey-Bass Publishers (1995)

Román, J. M. y S. Gallego, Acra: Escala de Estrategias de Aprendizaje, Manual ACRA, Publicaciones de Psicología Aplicada, 24-43, TEA Ediciones, Madrid (1994)

Trilling, B., y C. Fadel, 21st Century Skills: Learning for Life in Our Times, 30-75, John Wiley y Sons, San Francisco, EUA (2009)

Valencia-Serrano, M., J. Duarte-Soto y A.M. Caicedo-Tamayo, Aprendizaje Autorregulado, Metas Académicas y Rendimiento en Evaluaciones de Estudiantes Universitarios, ISSN: 1607-4041, Pensamiento Psicológico, 11 (2), 53-70 (2013)

Veas, A., J. A. López-López y otros tres autores, Differences in cognitive, motivational and contextual variables between under-achieving, normally-achieving, and over-achieving students: A mixed-effects analysis, doi: 10.7334/psicothema2016.283, Psicothema, 29 (4), 533-538 (2017)

Zimmerman, B.J., Motivational Sources and Outcomes of Self-Regulated Learning and Performance. En Zimmerman Handbook of self-regulation of learning and performance, Routledge, 49-64, Nueva York (2011) 